\title{
Emissions and economic costs of cycling compact fluorescent lamps with integrated ballasts
}

FG Rosillo

\author{
F Castejón
}

\author{
MA Egido
}

This paper proposes a way to quantify the emissions of mercury $(\mathrm{Hg})$ and $\mathrm{CO}_{2}$ associated with the manufacture and operation of compact fluorescent lamps with integrated ballasts (CFLis), as well as the economic cost of using them under different operating cycles. The main purpose of this paper is to find simple criteria for reducing the polluting emissions under consideration and the economic cost of CFLi to a minimum. A lifetime model is proposed that allows the emissions and costs to be described as a function of degradation from turning CFLi on and their continuous operation. An idealized model of a CFLi is defined that combines characteristics stated by different manufacturers. In addition, two CFLi models representing poor-quality products are analyzed. It was found that the emissions and costs per unit of time of operation of the CFLi depend linearly on the number of times per unit of time it is turned on and the time of continuous operation. The optimal conditions (lowest emissions and costs) depend on the place of manufacture, the place of operation and the quality of the components of the lamp/ballast. Finally, it was also found that for each lamp, there are intervals when it is turned off during which emissions of pollutants and costs are identical regardless of how often the lamp is turned on or the time it remains on. For $\mathrm{CO}_{2}$ emissions, the lamp must be off up to 5 minutes; for the cost, up to 7 minutes and for $\mathrm{Hg}$ emissions, up to 43 minutes. It is advisable not to turn on a CFLi sooner than $\mathbf{4 3}$ minutes from the last time it was turned off.

\section{Nomenclature}

a interval between the time the lamp is turned on and the next time it is turned off in a cycle

$b$ time that the lamp remains off in a cycle time the lamp remains off such that $\mathrm{CO}_{2}$ emissions are identical in any cycle

$b_{\text {opt }}^{\mathrm{Hg}}$ time the lamp remains off such that $\mathrm{Hg}$ emissions are identical in any cycle
$b_{\text {opt }}$ time the lamp remains off such that the costs are identical in any cycle
$C_{a, n} \quad$ cost per unit of time of the CFLi in cycling characterized by $(a, n)$
$C_{b, n} \quad$ cost per unit of time of the CFLi in cycling characterized by $(b, n)$
$C^{C^{\prime}}$ cost of electricity in the country in which the lamp is being used
$C_{I}$ cost of acquiring the lamp
$c_{m}$ mercury content dumped into the environ- ment per lamp
e $E / P$


$E$ number of particles emitted by the electrode each time it is turned on

$E_{\mathrm{CO}_{2}}^{c^{\prime}}$ amount of $\mathrm{CO}_{2}$ emitted by the electricity consumed in country $c^{\prime}$ where the CFLi is operating

$E_{\mathrm{CO}_{2}}^{c}$ amount of $\mathrm{CO}_{2}$ emitted by the electricity consumed in country $c$ where the CFLi is manufactured

$E_{a n}^{\mathrm{CO}_{2}} \quad \mathrm{CO}_{2}$ emissions during cycling characterized by $(a, n)$ per unit of time of reference

$E_{b, n}^{\mathrm{CO}_{2}} \quad \mathrm{CO}_{2}$ emissions during cycling characterized by $(b, n)$ per unit of time of reference

$E_{a n}^{H g} \quad \mathrm{Hg}$ emissions during cycling characterized by $(a, n)$ per unit of time of reference

$E_{b n}^{H g} \quad \mathrm{Hg}$ emissions during cycling characterized by $(b, n)$ per unit of time of reference

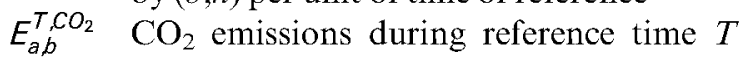
with CFLi operating in cycling characterized by $(a, b)$

f $F / P$

$F$ number of particles emitted by the electrode during normal operation per unit of time

$i$ discount rate

$l t_{\text {on }}$ lifetime of the lamp (the time the lamp remains on before it stops working)

$l t_{\text {on }+ \text { off }}$ lifetime of the lamp including the time that the lamp remains off

$L W F_{p}$ correction factor for power quality of $\mathrm{CFLi}$

$M C_{a, n}$ cost of acquisition with CFLi operating in cycling characterized by $(a, n)$

$M E_{a n}^{C_{2}} \quad \mathrm{CO}_{2}$ emissions from manufacturing the lamp with CFLi operating in cycling characterized by $(a, n)$

$M E_{a n}^{H g} \mathrm{Hg}$ emissions from manufacturing the lamp with CFLi operating in cycling characterized by $(a, n)$

$N$ number of starts during time $T$

$n$ number of times turned on per unit of time

$O C_{a, n}$ cost of operation with CFLi operating in cycling characterized by $(a, n)$

$O E_{a n}^{\mathrm{CO}_{2}} \quad \mathrm{CO}_{2}$ emissions from operating the lamp with CFLi operating in cycling characterized by $(a, n)$
$O E_{a n}^{H g} \mathrm{Hg}$ emissions from operating the lamp with CFLi operating in cycling characterized by $(a, n)$

$P$ number of particles susceptible to being detached from the electrode by sputtering or by evaporation

$P_{\text {elec }}(t)$ number of particles present in the electrode at time $t$

$P_{l}$ lamp power consumption

$P_{l p f}$ product of $P_{l}$ and $L W F_{p}$

$r_{c}$ portion of the CFLi that are used and returned to the recycle chain

$T$ reference time including on and off times

$T_{e}$ time in years the discount rate covers

$u_{m}$ energy consumed in manufacturing a CFLi

\section{Introduction}

The use of any technological device is associated with polluting emissions and economic costs. This study estimates the $\mathrm{CO}_{2}$ and mercury $(\mathrm{Hg})$ emissions of several compact fluorescent lamps with integrated ballasts (CFLis) and the economic cost associated with their lifecycle.

There is a widely spread mythology related to turning on and operating fluorescent lamps such as that turning on and off the lamp should be avoided because it consumes an exorbitant amount of energy. There are a limited number of studies that analyze in more or less detail the polluting emissions or the cost associated with the use of fluorescent lamps in general compared to other light source technologies. ${ }^{1-16}$ From the studies mentioned, it is difficult to extract any standards of use that enable CFLi emissions and costs to be made easily communicable to experts interested in illumination, much less to the final user.

This paper makes an estimation of some of the polluting emissions associated with CFLi and the economic cost of their use based on an easily understandable lifetime model which has been compared with experimental data. The calculations are made for the use of CFLi 
in Spain, discussing the parameters that could influence these calculations. Emissions during the production and use stages are included, attributing an amount of $\mathrm{Hg}$ to the production stage which is impossible to recover because many CFLi are disposed of outside the recycling chains. We attempt to compare the results with some of the recommendations made in previous studies and in manufacturers' technical documents. ${ }^{1,17}$ Finally, the results are used to provide a guide for the final user.

The case under study is residential application of the CFLi because of its social and educational interest. Furthermore, the choice of a CFLi instead of a compact lamp without integrated ballast or a fluorescent tube allows the whole problem to be dealt with, because in the CFLi, the fluorescent lamp itself and its ballast are inseparable. The emissions evaluated $\left(\mathrm{CO}_{2}\right.$ and $\left.\mathrm{Hg}\right)$ were chosen for their paradigmatic character, $\mathrm{CO}_{2}$ as an emission indicative of climate change and energy efficiency, and $\mathrm{Hg}$ because it is specifically associated with fluorescent lamps. It should be emphasized that the comparison made in this paper is between different ways of cycling CFLi and qualities but not different illumination technologies.

\section{Lifetime model}

In two studies similar to this, other authors have used an empirical model based on a Weibull distribution. ${ }^{1,15}$ Application of this distribution requires knowledge of three parameters whose relationship to the phenomena that influence lamp lifetime is not easy to establish. A lifetime model is proposed based on two parameters, one that describes the effect of turning them on and the other describing the effect of continuous operation. In spite of its simplicity and of consisting of only two parameters instead of three, the model proposed is based on physical parameters and provides fits of similar quality.
Before it starts to operate, the electrode of a fluorescent lamp is characterized by having a certain number of particles susceptible to being detached from the electrode by sputtering or by evaporation ${ }^{18}$ and able to emit electrons, which we shall call $P$. Each of these particles is defined as the average particle object of evaporation, sputtering, and in general, any physical phenomenon which causes loss of material from the electrode. To simplify, in the following, the average particle emitted by the electrode in any of the processes mentioned is assumed to be identical. Instantaneous variation of particles present in the electrode is given by the equation:

$$
\frac{\mathrm{d} P_{\text {elec }}(t)}{\mathrm{d} t}=F+\frac{E}{a}
$$

where $P_{\text {elec }}(t)$ represents the number of particles present in the electrode at time $t . P_{\text {elec }}(t)$ is a function of time, since the electrode undergoes the loss of material due to continuous operation and to start-up. According to this definition, $\mathrm{d} P_{\text {elec }}(t) / \mathrm{d} t$ represents the number of particles per unit of time emitted by the electrode at time $t$ due to different physical phenomena, $F$ represents the number of particles emitted by the electrode during normal operation per unit of time and $E$ is the number of particles emitted by the electrode each time it is turned on, and has been divided by $a$, the interval between the time the lamp is turned on and the next time it is turned off. The approach considers $F$ and $E$ constant throughout the lifetime of the lamp and characteristic of each ballast for a given fluorescent lamp. At any time $t$ after the first start-up, the following equation which describes the conservation of matter must be met:

$$
P=P_{\text {elec }}(t)+\int_{0}^{t}\left(F+\frac{E}{a}\right) \mathrm{d} t
$$


The lifetime of the lamp is designated by $l t_{o n}$. This number represents the time the lamp remains on before it stops working because the electrodes are exhausted. It should be stressed that this does not include the time the lamp remains off between start-ups. $P_{\text {elec }}\left(l t_{\text {on }}\right) \approx 0$, since at the end of its useful life, the lamp will have lost most of its electron emission coating. Substituting generic time $t$ in Equation (2) with lifetime $l t_{o n}$ and solving we have:

$$
l t_{o n}=\frac{P}{F+\frac{E}{a}}
$$

To solve Equation (3) from experimental data, since it is impossible to know $P$ explicitly, new variables $f=F / P$ and $e=E / P$ are defined with which Equation (3) can be rewritten in the following manner:

$$
l t_{\text {on }}=\frac{1}{f+\frac{e}{a}}
$$

The variables $f$ and $e$ supply measures of the reduction of lifetime due to normal operation and starting, respectively.

It is useful to define the lifetime of the lamp including time $b$ that the lamp remains off $l t_{\text {on }+ \text { off }}$ given by the following equation:

$$
l t_{o n+o f f}=\frac{a+b}{a} l t_{o n}
$$

This model coincides completely with a previous proposal by two authors of this article. ${ }^{19,20}$ Defining $l t_{0}$ as the nominal lifetime of the lamp, the variables used in the original model ( $A$ and $B$ ) are linearly related to those in the present proposal, in which the following relationships may be established immediately:

$$
\begin{gathered}
e=-\frac{A}{l t_{0}} \\
f=\frac{1-B}{l t_{0}}
\end{gathered}
$$

The advantage of the version of the lifetime model presented here is that it is based on physical principles down to the level of the particles that make up the electrodes and that understanding of the meaning of $e$ and $f$ is more direct than of $A$ and $B$.

Although this deduction was made considering the degradation mechanisms of fluorescent lamps, in a previous study, ${ }^{19}$ it was found that the fit of the model (based on $A$ and $B$ ) to experimental data available for $\mathrm{CFLi}^{21}$ was acceptable with an $R^{2}$ of 0.86 for the fit of experimental data and model. In this application, $f$ describes the effect of continuous operation during the lifetime of the lamp/ ballast pair, while $e$ does the same for turning it on.

\section{Equations describing emissions and costs}

$\mathrm{CO}_{2}$ emissions associated with the use of a CFLi are essentially produced by consumption of electricity during lamp production and during its operation. ${ }^{9}$ A reference time $T$ corresponds to the time a user keeps the CFLi in use, including both the time the lamp remains on and the time it remains off. $T$ can be compared to the lifetime in years that manufacturers assign to their $\mathrm{CFLi}$ in the basic technical documents directed at the final user for his orientation. 22

During time $T$, the lamp may break and have to be replaced. The lamp is subjected to cycling during which it remains on for time $a$ and off for time $b$. The time the lamp remains on when it is subjected to the cycling described is given by $a T /(a+b) . E_{a, b}^{T, C O_{2}}$ is defined as $\mathrm{CO}_{2}$ emissions during reference time $T$ with CFLi operating in cycling characterized by $(a, b)$ as given by the following equation:

$$
E_{a, b}^{T, \mathrm{CO}_{2}}=\frac{T}{l t_{o n+o f f}(a)} E_{\mathrm{CO}_{2}}^{c} u_{m}+\frac{a T}{a+b} E_{\mathrm{CO}_{2}}^{c^{\prime}} P_{l p f}
$$


where $l t_{\text {on }+ \text { off }}(a)$ is the lifetime, including time off, in cycling characterized by $a, E_{\mathrm{CO}_{2}}^{c}$ is the amount of $\mathrm{CO}_{2}$ emitted by the electricity consumed in country $c$ where the CFLi is manufactured, $u_{m}$ is the energy consumed in manufacturing a $\mathrm{CFLi}, E_{\mathrm{CO}_{2}}^{c^{\prime}}$ is the amount of $\mathrm{CO}_{2}$ emitted by the electricity consumed in country $c^{\prime}$ where the CFLi is operating. $P_{l p f}=P l L W F_{p}$ is the product of lamp power consumption $P_{l}$ and a correction factor for the CFLi power consumption quality, $L W F_{p}$, that accounts for the low power factor of $\mathrm{CFLi} .{ }^{16}$ In this study, a value of 1.05 is assigned to $L W F_{p}$ that represents a $5 \%$ increase of $P_{l}{ }^{16} T /$ $l_{\text {on }+ \text { off }}(a)$ is the number of CFLi used during time $T$, and the first term in Equation (8) shows the emissions from manufacturing the number of lamps necessary to provide service during time $T$. Taking into account that $a T /(a+b)$ is the time that the CFLi remains on during time $T$, the second term gives the emissions associated with CFLi operation.

As the number of starts during time $T$ is given by $N=T /(a+b), n$ is defined as the number of times turned on per unit of time:

$$
n=\frac{N}{T}=\frac{1}{a+b}
$$

This study evaluates the emissions and costs by normalizing the unit of time $T$, so that $E_{a, n}^{\mathrm{CO}_{2}}=E_{a, b}^{T, \mathrm{CO}_{2}} / T$ are defined as the $\mathrm{CO}_{2}$ emissions during cycling characterized by $(a, n)$ per unit of time of reference. Taking into account Equations (4) and (8), it is expressed as follows:

$$
E_{a, n}^{\mathrm{CO}_{2}}=a n\left(E_{\mathrm{CO}_{2}}^{c} u_{m}\left(f+\frac{e}{a}\right)+E_{\mathrm{CO}_{2}}^{c^{\prime}} P_{l p f}\right)
$$

The relationship between $\mathrm{CO}_{2}$ emissions from manufacturing the lamp $M E_{a, n}^{C O_{2}}$ and from its operation $\mathrm{OE}_{a, n} \mathrm{CO}_{2}$ may be evaluated as:

$$
\frac{M E_{a, n}^{C O_{2}}}{O E_{a, n}^{C O_{2}}}=\frac{E_{C O_{2}}^{c} u_{m}}{E_{\mathrm{CO}_{2}}^{c^{\prime}} P_{l p f}}\left(f+\frac{e}{a}\right)
$$

Based on Equation (10), and keeping in mind that $a=1 / n-b$, making $E_{a_{2} n}^{\mathrm{CO}_{2}}$ the same for any $n$, and with a few operations, a time may be found when the lamp remains off, $b_{\text {opt }}{ }^{\mathrm{CO}_{2}}$, such that the emissions are identical in any cycle, regardless of the time on, $a$, and the number of starts per unit of time, $n$ :

$$
b_{o p t}^{C O_{2}}=\frac{e E_{\mathrm{CO}_{2}}^{c} u_{m}}{E_{\mathrm{CO}_{2}}^{c^{\prime}} P_{l p f}+f E_{\mathrm{CO}_{2}}^{c} U_{m}}
$$

A version of Equation (10) may also be used in which $a$ is substituted by $b$ through the expression $a=1 / n-b$ called $E_{b, n}^{C O_{2}}$.

The deduction and equations for $\mathrm{Hg}$ emissions $E_{a, n}^{H g}$ and $M E_{a, n}^{H g} / O E_{a, n}^{H g}$ are similar. In this case, a portion $r_{c}$ of the CFLi that are used are returned to the recycle chain so that a portion $1-r_{c}$ and its $\mathrm{Hg}$ content $c_{m}$ is dumped into the environment. Under these conditions, the equations are the following:

$$
\begin{gathered}
E_{a, n}^{H G}=a n\left(\left(E_{H g}^{c} u_{m}=c_{m}\left(1-r_{c}\right)\right)\left(f+\frac{e}{a}\right)\right. \\
\left.+E_{H g}^{c^{\prime}} P_{l p f}\right) \\
\frac{M E_{a, n}^{H g}}{O E_{a, n}^{H g}}=\frac{E_{H g}^{c} u_{m}+c_{m}\left(1-r_{c}\right)}{E_{H g}^{c^{\prime}} P_{l p f}}\left(f+\frac{e}{a}\right)
\end{gathered}
$$

and

$$
b_{o p t}^{H g}=\frac{e\left(E_{H g}^{c} u_{m}+c_{m}\left(1-r_{c}\right)\right)}{E_{H g}^{c^{\prime}} P_{l p g}+f\left(E_{H g}^{c} u_{m}+c_{m}\left(1-r_{c}\right)\right)}
$$


It should be emphasized that the accounting of emissions from manufacturing $M E_{a, n}^{H g}$ includes both $\mathrm{Hg}$ emitted by consumption of electricity during lamp manufacture from fuels used for generating electricity, and the $\mathrm{Hg}$ in the composition of gas in the fluorescent lamp not recycled.

Finally, the equations and concepts are analogous for the economic cost to the final user. $C_{a, n}$ is defined as the cost per unit of time of the CFLi in cycling characterized by $(a, n)$ :

$$
C_{a, n}=a n\left(C_{l}\left(f+\frac{e}{a}\right)+C^{C^{\prime}} P_{l p f}\right)
$$

where $C_{l}$ is the cost of acquiring the lamp and $C^{C^{\prime}}$ the cost of electricity in the country in which it is being used. In a somewhat different manner, a similar economic assessment was previously developed by Carriere and Rea, ${ }^{1}$ with the main difference being that in the study cited, the lifetime was evaluated based on a Weibull distribution. Following the cost assessment methodology that includes the discount rate $i$ as an annual percentage, and $T_{e}$, the time in years the discount rate covers and which allows the value of money to be updated to the present time, ${ }^{4,5}$ a somewhat more complicated expression is used:

$$
\begin{aligned}
C_{a . n}= & a n\left(C_{l}\left(f+\frac{e}{a}\right)\left(1+\frac{i}{100}\right)^{T_{e}}\right. \\
& \left.+C^{C^{\prime}} P_{l p f} \frac{\left(1+\frac{i}{100}\right)^{T_{e}}-1}{\frac{i}{100}}\right)
\end{aligned}
$$

The ratio between the cost of acquisition $M C_{a, n}$ and the cost of operation $O C_{a, n}$ of the lamp is given by the following equation:

$$
\frac{M C_{a, n}}{O C_{a, n}}=\frac{C_{l}}{C^{C} P_{l p f}}\left(f+\frac{e}{a}\right) \frac{\left(1+\frac{i}{100}\right)^{T_{e}}}{\left(1+\frac{i}{100}\right)^{T_{e}}-1} \frac{100}{i}
$$

If the discount rate is not considered, we have:

$$
\frac{M C_{a, n}}{O C_{a, n}}=\frac{C_{l}}{C^{C} P_{l p f}}\left(f+\frac{e}{a}\right)
$$

The optimal economic value of the time off $b_{\text {opt }}$ is given by:

$$
b_{o p t}=\frac{e C_{l}\left(1+\frac{i}{100}\right)^{T_{e}}}{C^{C^{\prime}} P_{l p f} \frac{\left(1+\frac{i}{10)^{T_{e}}}-1\right.}{\frac{i}{100}}+f C_{l}\left(1+\frac{i}{100}\right)^{T_{e}}}
$$

Or, if the discount rate is ignored:

$$
b_{o p t}=\frac{e C_{l}}{C^{C^{t}} P_{l p f}+f C_{l}}
$$

It is possible to evaluate the emissions and cost per lumen-hours ${ }^{2}$ just by dividing Equations (10), (13) and (16), respectively, by the integral of the luminous flux generated during CFLi lifetime. In general, this calculation would involve the time integration due to the reduction of the luminous flux emitted during lamp lifetime (lumen maintenance). This parameter is especially useful for performing comparisons of different technologies as, for example, CFLi, incandescent and light-emitting diodes (LEDs) and for comparison of models of the same technology. ${ }^{2}$ However, it does not supply simple rules of use to the final user. The purpose of the present work is not to calculate this quantity but to investigate rules that make it possible to evaluate selected scenarios of use of some models of CFLi that supply a criterion for switching on and off the lamps, independently of the cost and emission per lumen generated for the lamp during its life. For this reason, this quantity has not been evaluated in the present work. On the other hand, a simple estimation of this quantity can be performed 
easily with the data supplied here, as will be stated in the chapter of results.

\section{CFLi model}

There is no complete set of data related to a specific CFLi that makes this study possible. It was decided to define an ideal fluorescent lamp model that combines manufacturers' data for similar models in an average range of CFLi qualities. Table 1 shows the data used for the CFLi model. The CFLi described does not exist, but it is also true that it could perfectly well exist and be equivalent to one of the models found in major manufacturers' catalogues.

The origins of the data selected are specified below. The model proposed is considered a good-quality CFLi in the average commercial range of features and prices. It is usually possible to find the lifetime of a CFLi in the conventional 2 hours 45 minutes on/ 15 minutes off (or similar) test. But to infer $e$ and $f$ from Equation (4), the lifetime in at least two different cycles must be known. The $e$ and $f$ in Table 1 were calculated based on the manufacturer's data for the CFLi. ${ }^{23} \mathrm{CFLi} \mathrm{Hg}$ content estimates by manufacturers differ $3,8,10,24-28$ from $1 \mathrm{mg}$ to $27 \mathrm{mg}$ of $\mathrm{Hg}$ per $\mathrm{CFLi}$, which makes it difficult to select one concrete value. It was decided to choose one for the CFLi quality considered with low $\mathrm{Hg}$ content, but somewhat higher than the minimums stated for that quality of lamp. Electricity used in manufacturing the lamp somewhat higher than the most similar reference was taken for a $15 \mathrm{~W} \mathrm{CFLi}{ }^{9,29}$ Finally, we took the rounded price of a CFLi with consumption and lifetime described for Spain according to prices published by one of the major manufacturers. ${ }^{30}$

Consumption of $18 \mathrm{~W}$ was selected as equivalent to the $100 \mathrm{~W}$ incandescent lamp commonly used in Spain and prohibited by European regulation in 2009. ${ }^{31}$ As described elsewhere, ${ }^{16}$ a correction factor for power quality, $L W F_{p}$ that accounts for the CFLi low-power factor must be considered.

The luminous flux of the model of CFLi described is $10001 \mathrm{~m}$, a value in the lower range of the luminous flux of real CFLi of a similar category.

In addition to the model described, which is a state-of-the-art good-quality CFLi (G Start-G Cont.oper), another two models of CFLi are used: One is poor quality (B Start-B Cont. oper) and one very poor (VB Start-VB Cont.oper). The three models are characterized by having different $e, f$ and price. Reference 21 shows that there are CFLi with much shorter lifetimes than the nominal. As we have no additional data, it is assumed that the rest of the characteristics (power consumed, $\mathrm{Hg}$ content, correction factor for power quality, electricity in manufacture and luminous flux) are identical in all the samples. Although this approximation could seem questionable, it happens to be useful in order to perform an initial approach to the problem. For the two poor-quality models, lower prices and higher $e$ and $f$ are proposed,

Table 1 Characteristics of the CFLi model analyzed in this study.

\begin{tabular}{ll}
\hline Power consumed $P_{I}$ & $18 \mathrm{~W}$ \\
Purchase price $C_{l}$ & 10 Euro \\
Lifetime/a & $10000 \mathrm{~h} / 3 \mathrm{~h}$ and $12500 \mathrm{~h} / 12 \mathrm{~h}$ \\
$e$ & $8.000 \mathrm{E}-05$ \\
$f$ & $2.037 \mathrm{E}-08\left(\mathrm{~s}^{-1}\right)$ \\
Hg content $c_{m}$ & $3 \mathrm{mg}$ \\
Electricity in manufacture $u_{m}$ & $4 \mathrm{kWh}$ \\
\hline
\end{tabular}

CFLi: compact fluorescent lamps with integrated ballasts; $\mathrm{Hg}$ : mercury. 
which result in a reduction of lifetime (Figure 1). Table 2 shows the data for the three models analyzed in this study. In the case of the two poor-quality lamps, prices are typical of shops in Madrid. The reference model is called $\mathrm{G}$ and the poor-quality and very poorquality models are called $\mathrm{B}$ and VB, respectively.

Figure 1 shows the lifetime of the three models of CFLi compared to continuous operation time $a$ and the manufacturer's data, which make it possible to determine the coefficients of lifetime $e$ and $f$ for the good-quality model.

\section{Description of cycling}

It was decided to evaluate the magnitudes considered for an arbitrary time $T$, which includes both the time the CFLi remain on and the time they are off. Cycles are defined by the number of times turned on per unit of time designated by $n\left(\mathrm{~h}^{-1}\right)$, by time $a(m)$ that the lamp remains on between the time it is turned on and the following time it is turned off, and finally by time $b(m)$ that the lamp is off between the time it is turned off and the following time it is turned on. The real time of operation when the CFLi is on is given by

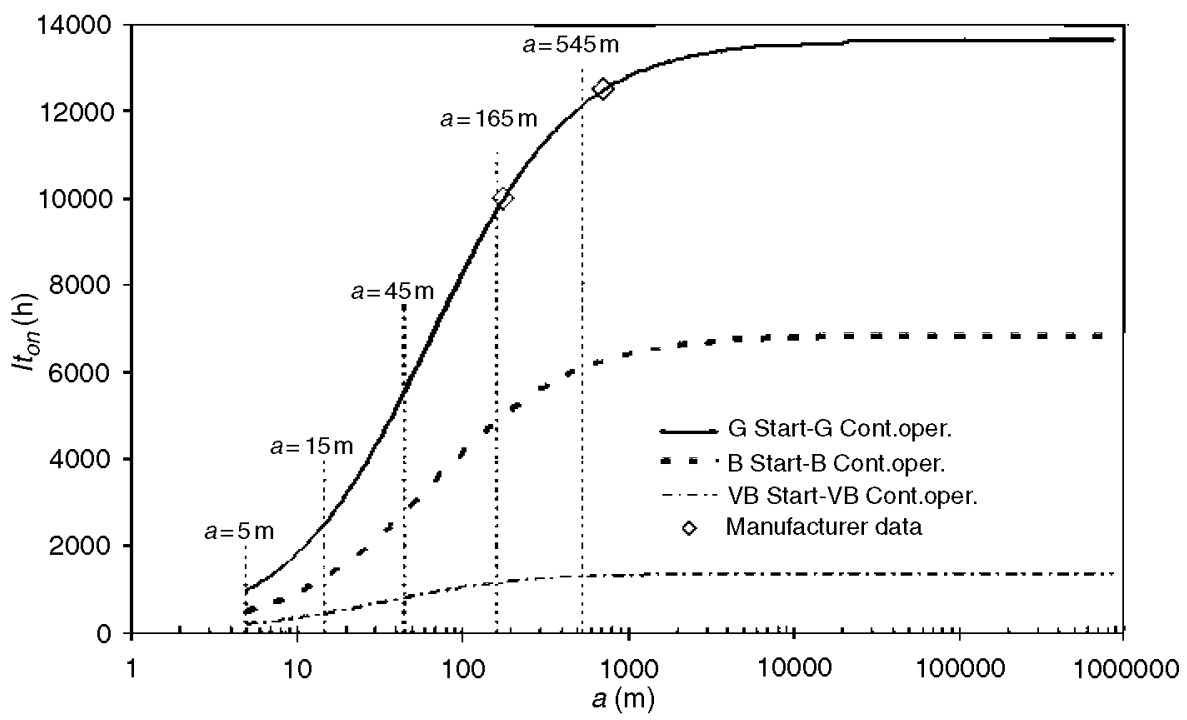

Figure 1 Compact fluorescent lamps with integrated ballasts (CFLi) lifetime data according to the manufacturer's technical specification sheets. The lifetime of a good-quality CFLi model (G Start-G Cont.oper) poor-quality CFLi model (B Start-B Cont.oper) and very poor-quality CFLi model (VB Start-VB Cont. oper) for different a. The vertical dotted lines show several a used to make some of the estimates

Table 2 Prices and coefficients that determine the lifetime of CFLi models analyzed

\begin{tabular}{llll}
\hline Model & Purchase price $C_{l}$ (Euro) & $e$ & $f\left(\mathrm{~s}^{-1}\right)$ \\
\hline $\mathrm{G}$ & 10 & $8.000 \mathrm{E}-05$ & $2.037 \mathrm{E}-08$ \\
$\mathrm{~B}$ & 6 & $1.600 \mathrm{E}-04$ & $4.074 \mathrm{E}-08$ \\
VB & 3 & $4.000 \mathrm{E}-04$ & $2.037 \mathrm{E}-07$ \\
\hline
\end{tabular}

CFLi: compact fluorescent lamps with integrated ballasts. 
$a T /(a+b)$. In homes, CFLis are turned off at night, which is equivalent to every 24 hours, one of the periods characterized by $b$ is longer than the value computed. This really has no effect on the calculations of interest for this study, except if it is necessary to estimate the time in years the user is going to use the CFLi. It can be demonstrated that if $b^{\prime}$ is the time that the CFLi remains off every night, that is every 24 hours, the ratio between the computation time $T$ in the cycle $(a, b)$ and the computation time $T^{\prime}$ in the cycle $\left(a, b, b^{\prime}\right)$ is given by $T^{\prime}=T\left(1+\left(b^{\prime}-b\right) /((a+b) 24 \times 60)\right)$ with $a, b$ and $b^{\prime}$ expressed in minutes.

Figure 2 shows graphically some of the cycles for which the polluting emissions and the economic cost were estimated. Each cycle is repeated until the end of time $T$, although it might be necessary to replace a CFLi that breaks, in which case, the emissions from manufacturing the new lamp have to be added. $b=15$ minutes in the first cycle up to cycle $n=3 / 1 \mathrm{~h}^{-1}$. For example, cycle $n=1 / 9 \mathrm{~h}^{-1}$ (that is turned on every 9 hours) can correspond to $a=525$ minutes as shown in Figure 2, but also to any $a$, as long as $a=1 / n-b$, for example, $a=240$ minutes and $b=300$ minutes.

No attempt was made to identify the cycles with real use profiles, which would have been less demanding, but to cover a wide range of cycles that make it possible to understand what happens in very different cycles. The extreme cycles of being turned on nine times per hour and of being turned on once every 9 hours provide a wide range of possibilities.

\section{Emission and cost framework}

It is assumed that the lamps used in Spain may have been manufactured in Germany or Slovakia. This assumption is based on checking shops in the city of Madrid and in the manufacturer's reference to the place of manufacture of his lamps. ${ }^{32}$

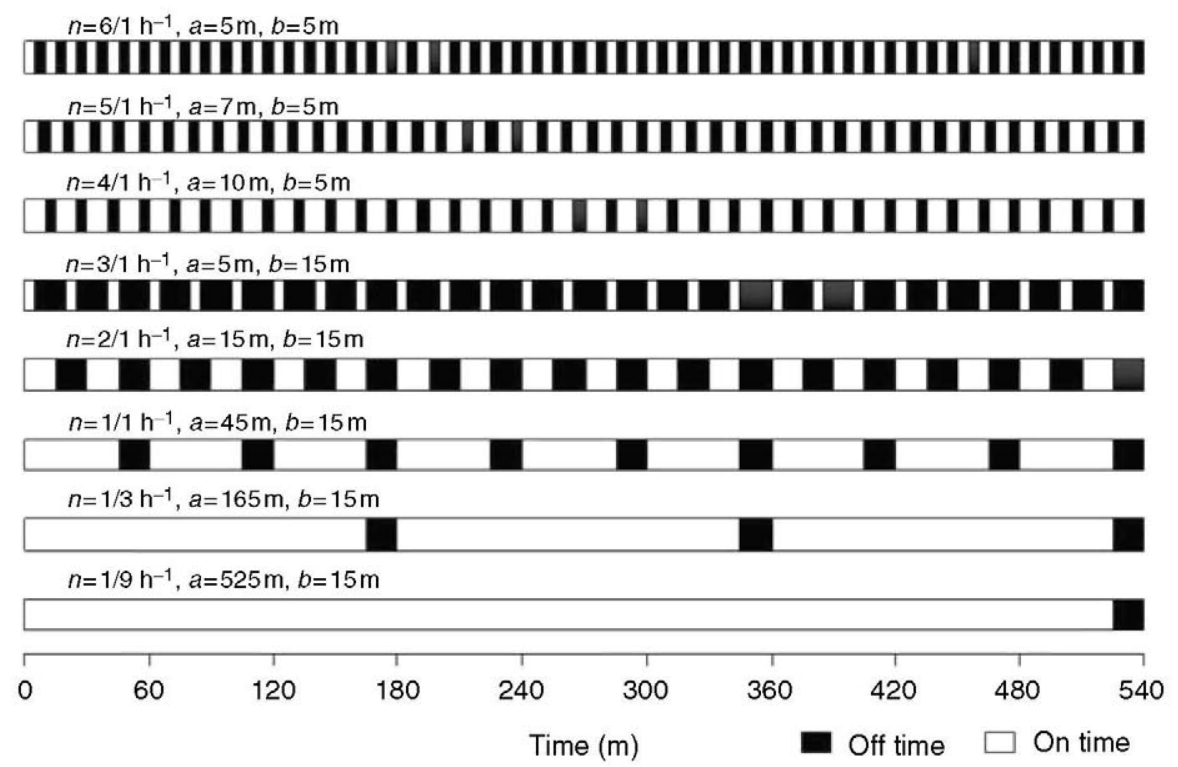

Figure 2 Diagram of some of the lifecycles for which the polluting emissions and economic cost of compact fluorescent lamps with integrated ballasts (CFLi) are estimated. $b=15$ minutes up to cycle $n=3 / 1 \mathrm{~h}^{-1}$, for faster cycles, $b=5$ minutes 
Table 3 shows the $\mathrm{CO}_{2}$ equivalent and $\mathrm{Hg}$ emissions from electricity consumed in Germany, Slovakia and $\operatorname{Spain}^{33}$ for 2007. Lower emissions are observed in Slovakia because of the higher component of nuclear energy in the electricity mix. The costs of radioactive waste management or safety problems are not evaluated in this study. In Germany and Slovakia, emissions are from manufacture of the lamp, while in Spain emissions are due to the electricity consumed during lamp use.

With reference to $\mathrm{CO}_{2}$ equivalent, the data used correspond to a Life Cycle Assessment (LCA) emission factors assessment scheme, which evaluates all the emissions generated for the production of the lamp and the consumption of electricity, and that include not only the $\mathrm{CO}_{2}$ emissions but also the equivalent ones due to the emissions of $\mathrm{CH}_{4}$ and $\mathrm{N}_{2} \mathrm{O}$. Further details of the methodology and origin of data can be encountered in Reference 33, the source of the data presented in Table 3.

To simplify, it is assumed that recycled lamps do not emit the $\mathrm{Hg}$ in the gas into the environment and that, on the contrary, lamps not recycled end up emitting all of the $\mathrm{Hg}$ that was originally contained in the gas into the environment. Table 3 shows an estimate of the number of residential CFLi recycled in Spain, which only comes to 2 out of 10 lamps. ${ }^{34}$ The price of electricity considered for Spain is for the residential sector and the year of reference is $2008 .^{35}$

The selection of the discount rate $i$ and computation time $T_{e}$ is essentially an arbitrary exercise. The discount rate is especially subject to economic fluctuations difficult to forecast that often depend on international events such as the attack of September 11th. It is estimated that the value of $i$ is between $7 \%$ and $12 \% .^{4-6}$ This study considers an annual discount rate of $7 \%$ for 3 years. The choice of 3 years for $T_{e}$ is based on the 3-year replacement warranty for broken CFLi given by manufacturers of CFLi, such as those analyzed in this study. ${ }^{22}$ Further below, $b_{\text {opt }}$ is computed for $12 \%$ discount rates and periods up to 10 years. $b_{\text {opt }}$ is also found without considering the discount rate and is expressed by Equation (21).

\section{Results and discussion}

\subsection{Emissions and costs of manufacture and operation}

Figures 3 and 4 present the ratio of $\mathrm{CO}_{2}$ equivalent and $\mathrm{Hg}$ emissions from $\mathrm{CFLi}$ manufacturing to emissions from operation,

Table $3 \quad \mathrm{CO}_{2}$ equivalent emissions $\left(E_{\mathrm{CO}_{2}}^{c}\right)$ and $\mathrm{Hg}\left(E_{\mathrm{Hg}}^{c}\right)$ from electricity consumption in countries of manufacture (Germany and Slovakia) and operation (Spain) of the CFLi. Portion of lamps recycled $\left(r_{c}\right)$ and price of electricity $\left(C_{c}\right)$ in Spain. Discount rate $(I)$ and time considered $\left(T_{e}\right)$ for the economic calculations

\begin{tabular}{lll}
\hline & Emissions from electricity consumption & \\
\cline { 2 - 3 } Country & $E_{\mathrm{CO}_{2}}^{c}\left(t \mathrm{CO}_{2} \mathrm{equ} / \mathrm{MWh}\right)$ & $E_{\mathrm{Hg}}^{c}(\mathrm{kgHg} / \mathrm{kWh})$ \\
\hline Spain & 0.639 & $9.55 \mathrm{E}-09$ \\
Germany & 0.706 & $3.02 \mathrm{E}-09$ \\
Slovakia & 0.353 & $7.60 \mathrm{E}-10$ \\
& Portion of CFLi recycled $r_{c}$ & Price of electricity $C^{c}$ (c Euro/kWh) \\
\hline Spain & 0.2 & 15.6 \\
& Discount rate, $i(\%)$ & Period of discount rate, $T_{e}$ (vears) \\
\hline
\end{tabular}

CFLi: compact fluorescent lamps with integrated ballasts; $\mathrm{Hg}$ : mercury. 


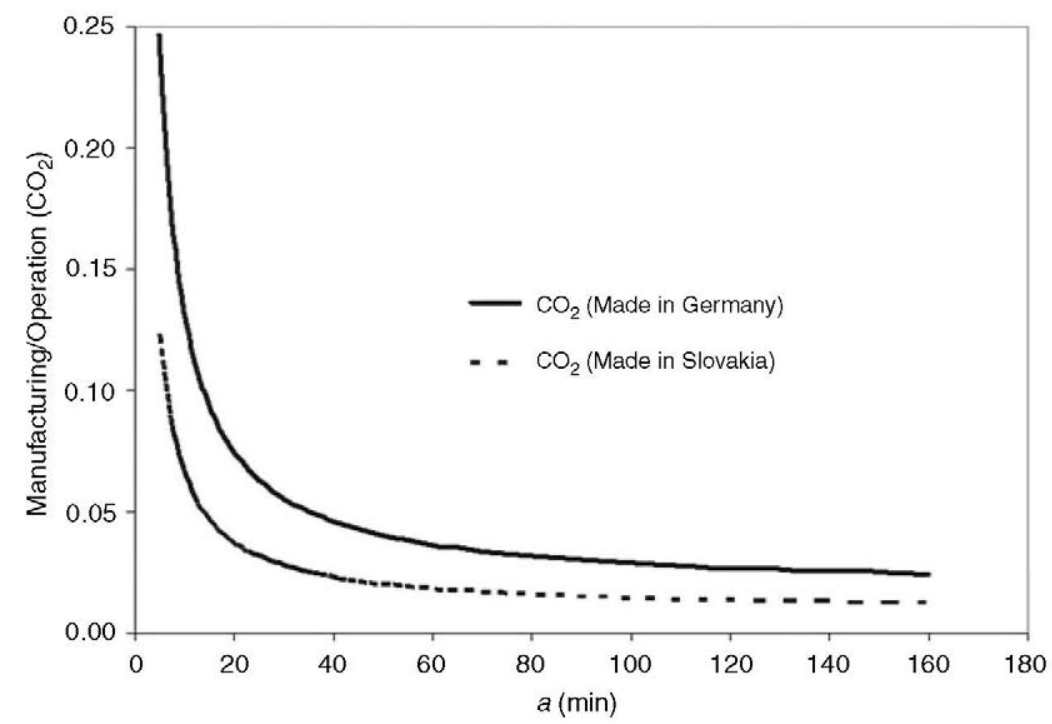

Figure 3 Ratio of $\mathrm{CO}_{2}$ equivalent emissions from manufacturing and from operation over time of continuous operation of the compact fluorescent lamps with integrated ballasts (CFLi)

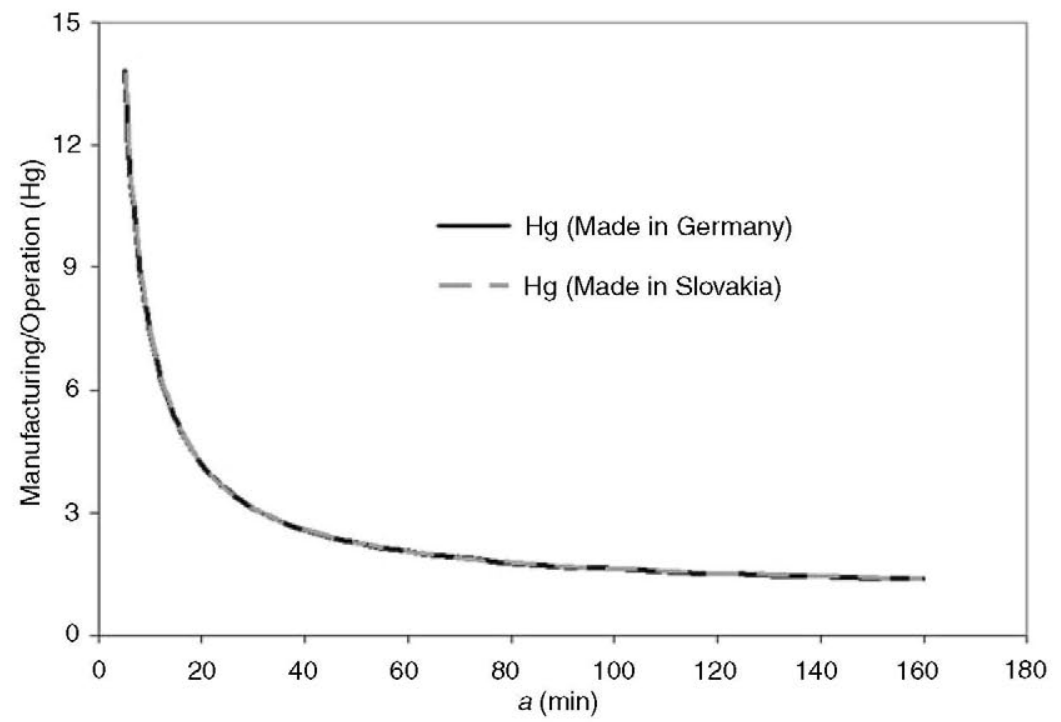

Figure 4 Rate of mercury $(\mathrm{Hg})$ emissions from manufacture and from operation over time of continuous operation of the compact fluorescent lamps with integrated ballasts (CFLi)

over time of continuous operation $a . \mathrm{Hg}$ Since the electricity mix in Germany is more emissions are accounted as manufacturing coal-intensive than in Slovakia, a lamp emissions. manufactured in Germany emits more $\mathrm{CO}_{2}$

In the case of $\mathrm{CO}_{2}$ equivalent emissions, equivalent during manufacturing than one operation predominates over manufacturing. manufactured in Slovakia. However, as the 


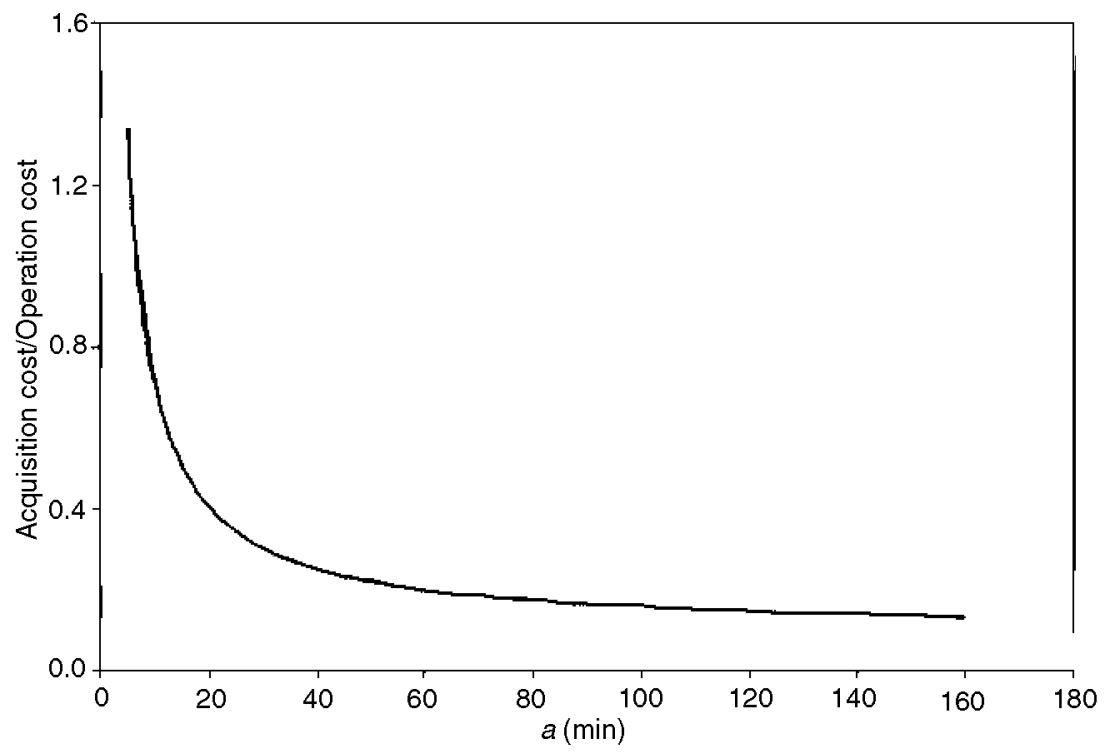

Figure 5 Ratio of the purchase price to the cost of operation at different continuous operation times, a, of the compact fluorescent lamps with integrated ballasts (CFLi)

emissions from operation in Spain are higher in both cases, the total $\mathrm{CO}_{2}$ equivalent emissions are similar, regardless of the country manufactured or the value of $a$.

In the case of $\mathrm{Hg}$ emissions, the situation is the contrary: As a consequence of the original $\mathrm{Hg}$ content in the CFLi and its low recuperation rate in Spain, the $\mathrm{Hg}$ emissions due to manufacturing clearly dominate over operation. Only at high $a$ are the emissions due to operation an appreciable portion of the total emissions.

The purchase price of the lamp represents an important part of the total cost of the CFLi. Both costs are equivalent for an $a$ near 7 minutes. Above this, cost of operation gains weight, and below it, purchase price gains weight as may be observed in Figure 5. If the discount rate had not been considered and Equation (19) had been used, $a$ at which the cost of operation and the purchase price are identical would be around 22 minutes.

\subsection{Influence of the number of times turned on and the time the CFLi remains on}

Figures 6 and 7 show the $\mathrm{CO}_{2}$ equivalent and $\mathrm{Hg}$ emissions per hour over the time of continuous operation, $a$, of the CFLi. The data given are for a lamp manufactured in Germany and purchased and used in Spain, as the figures for a lamp manufactured in Slovakia are similar.

The emissions during the lifecycle are proportional to $n$ as seen in Equations (10) and (13) and in Figure 6. There is also a term that depends on the product an. It is therefore impossible to find an ideal situation for reduction of emissions and costs, except if the CFLi are subjected to the lowest operation time possible with the minimum number of times turned on.

This extreme would be the absurd situation of not using the lamp. However, neither would it be reasonable to assume that leaving the lamp on is a good option. As an example, and insofar as $\mathrm{CO}_{2}$ emissions are concerned, 


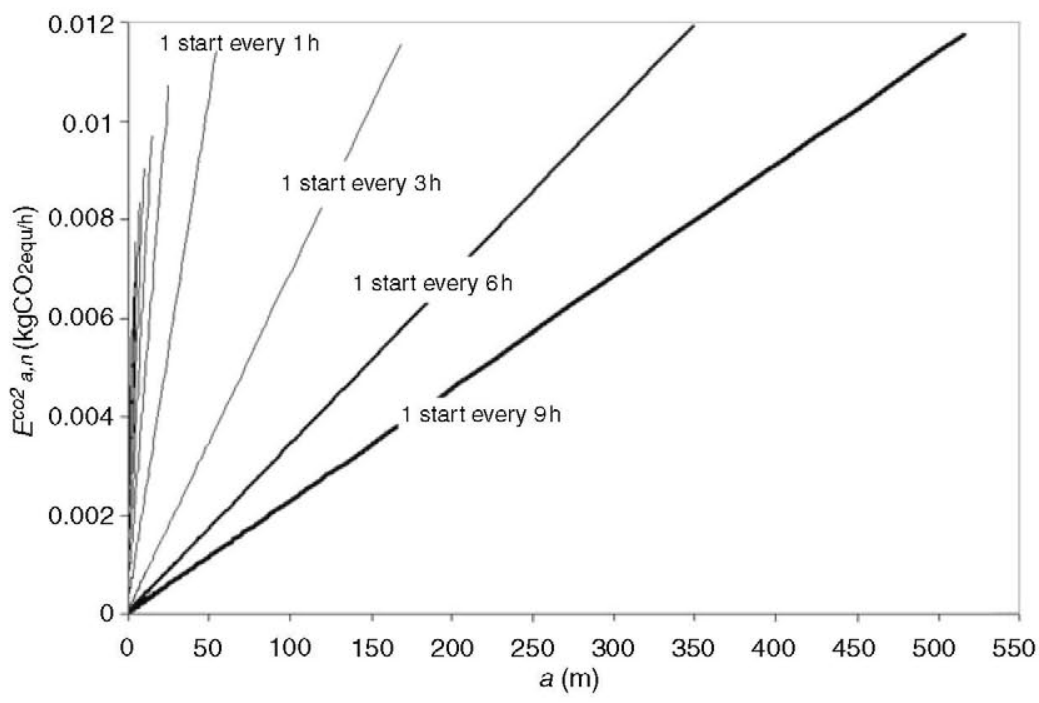

Figure $6 \mathrm{CO}_{2}$ emissions for a compact fluorescent lamps with integrated ballasts (CFLi) manufactured in Germany in operation in Spain over operating time

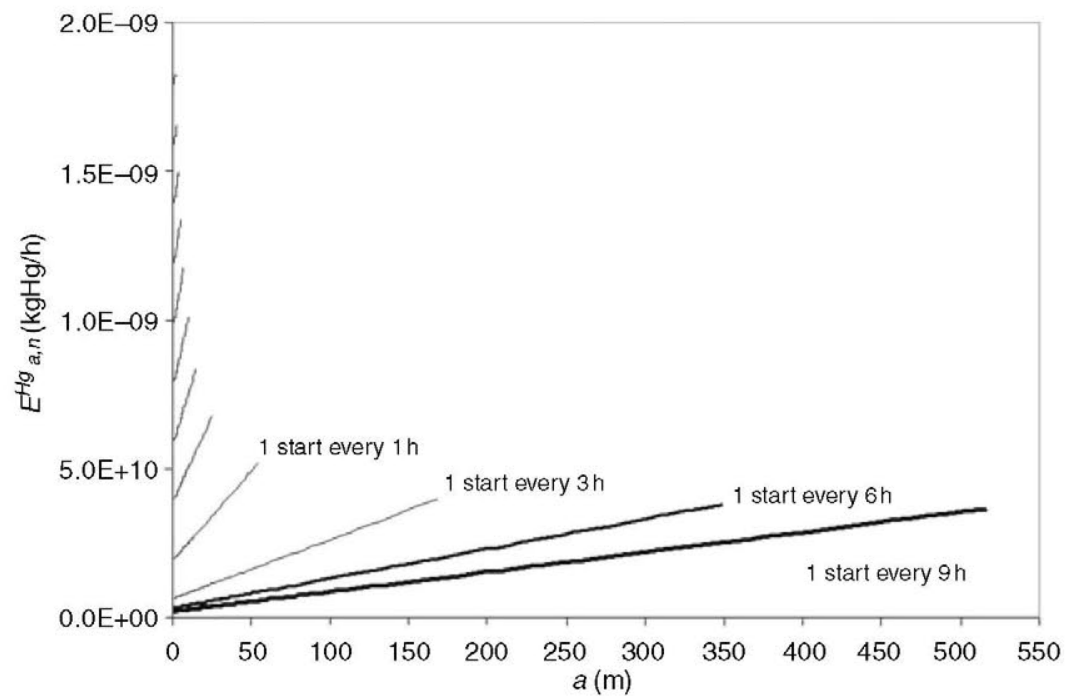

Figure 7 Mercury $(\mathrm{Hg})$ emissions for a compact fluorescent lamps with integrated ballasts (CFLi) manufactured in Germany in operation in Spain over time of operation

if the user is going to devote an hour to reading, it is not reasonable for the lamp to stay on longer. Cycling with starts once every 6 hours with an $a$ below 60 minutes means lower emissions than leaving it on for a longer time. Concerning the number of times turned on, the cycle in which it is turned on once every 3 hours with an $a$ of 60 minutes will always have higher $\mathrm{CO}_{2}$ emissions than the cycle before. Therefore, the valid criterion for 


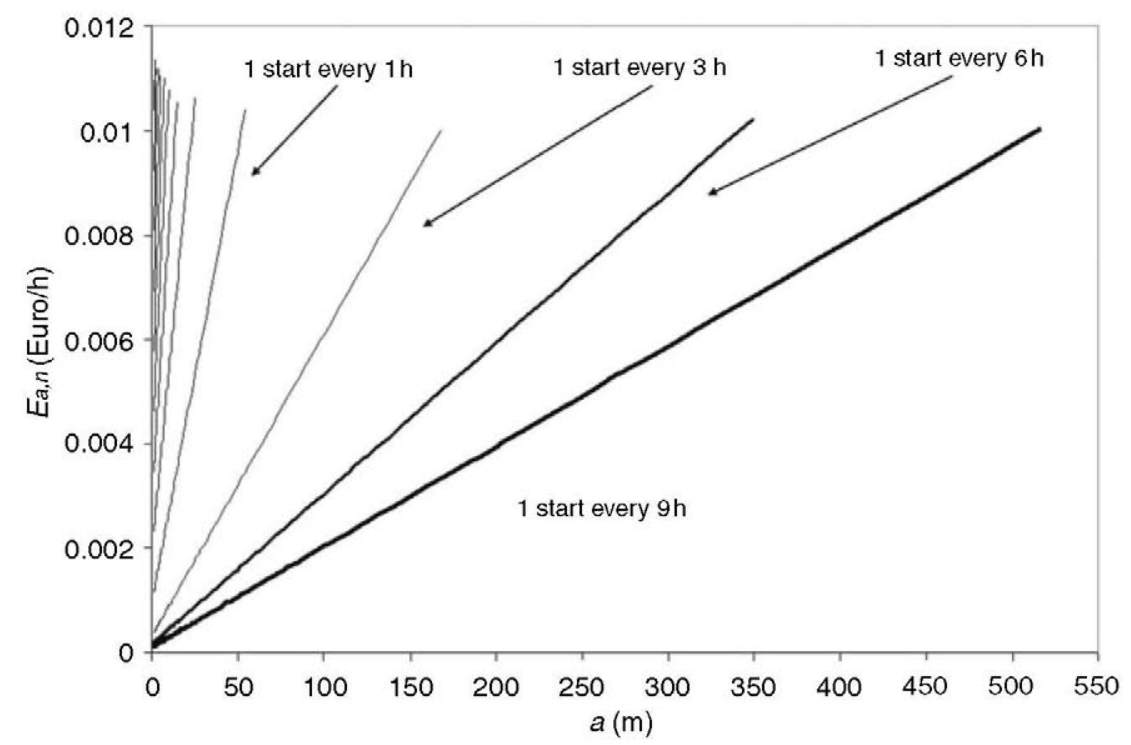

Figure 8 Costs of a compact fluorescent lamps with integrated ballasts (CFLi) manufactured in Germany or Slovakia in operation in Spain over time of operation

reducing $\mathrm{CO}_{2}$ emissions is to use the lamp when needed, turning it on a minimum number of times and for the shortest operating time possible.

The $\mathrm{Hg}$ emissions depend more strongly on the lifetime of the lamp due to the high original content in the CFLi, and therefore, on the number of times turned on. Figure 7 shows that, compared to $\mathrm{CO}_{2}$ emissions where all the lines converge practically at zero emissions at very low $a$ (Figure 6), the lines that represent $\mathrm{Hg}$ emissions for very low operation times, $a$, are scaled on the ordinate axis and do not converge at zero emissions.

The cost of the lamp is reflected in Figure 8 and shows intermediate behaviour between $\mathrm{CO}_{2}$ and $\mathrm{Hg}$ emissions: The purchase price has an important role starting at an $a$ of 7 minutes, so the cost lines are scaled similar to $\mathrm{Hg}$ but less exaggerated. The number of starts and the consequent reduction in lifetime of the lamp increase the cost noticeably, especially for $a$ under 7 minutes.
As it is not possible for the residential user to predict the cycling he is going to subject the CFLi to, or for him to have a calculator at hand to check whether it is worthwhile to turn off the lamps, the only valid criteria that makes common sense is to minimize the number of starts and the time the lamp is in operation, as long as it still fits the need for illumination. Only in the case of a business or industry with formal hours when it has to remain on in a room or workplace would it be possible to establish a more specific criterion of use.

Furthermore, while a higher number of starts leads to higher $\mathrm{Hg}$ emissions and economic costs due to the reduction in lifetime of the sample and the need for its replacement, a longer continuous operation time $a$ has a similar effect on $\mathrm{CO}_{2}$ emissions.

\subsection{Optimal time off}

The analysis of data up to now can hardly be made use of by the final user beyond what 


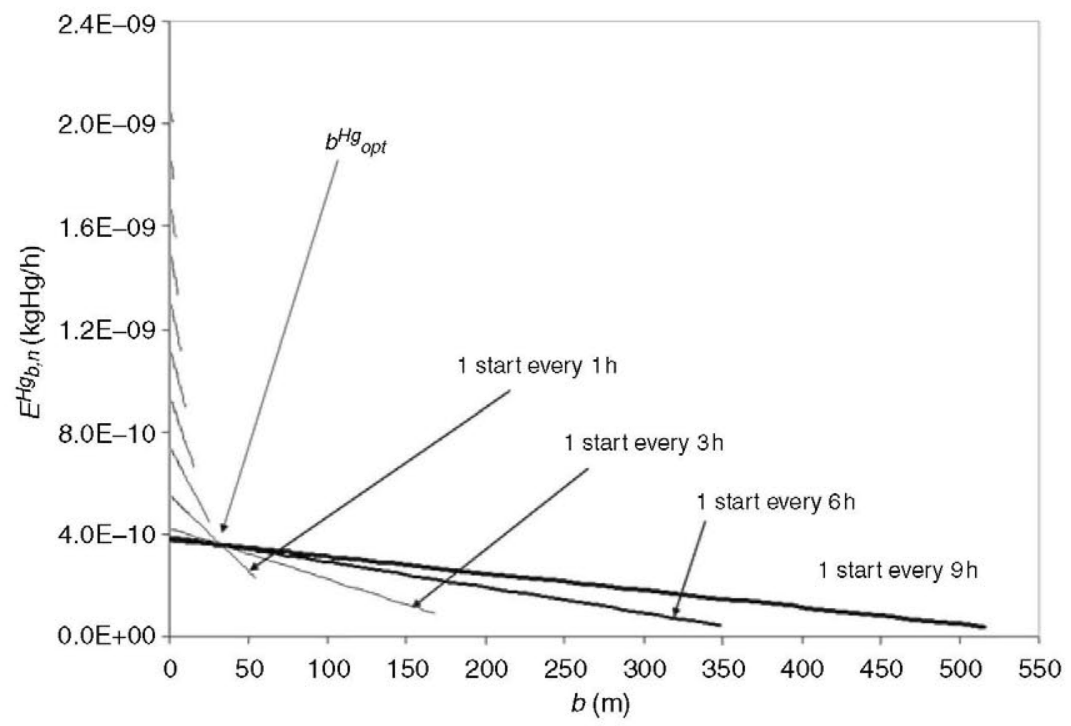

Figure 9 Mercury $(\mathrm{Hg})$ emissions for a compact fluorescent lamps with integrated ballasts (CFLi) manufactured in Germany and in operation in Spain over time off

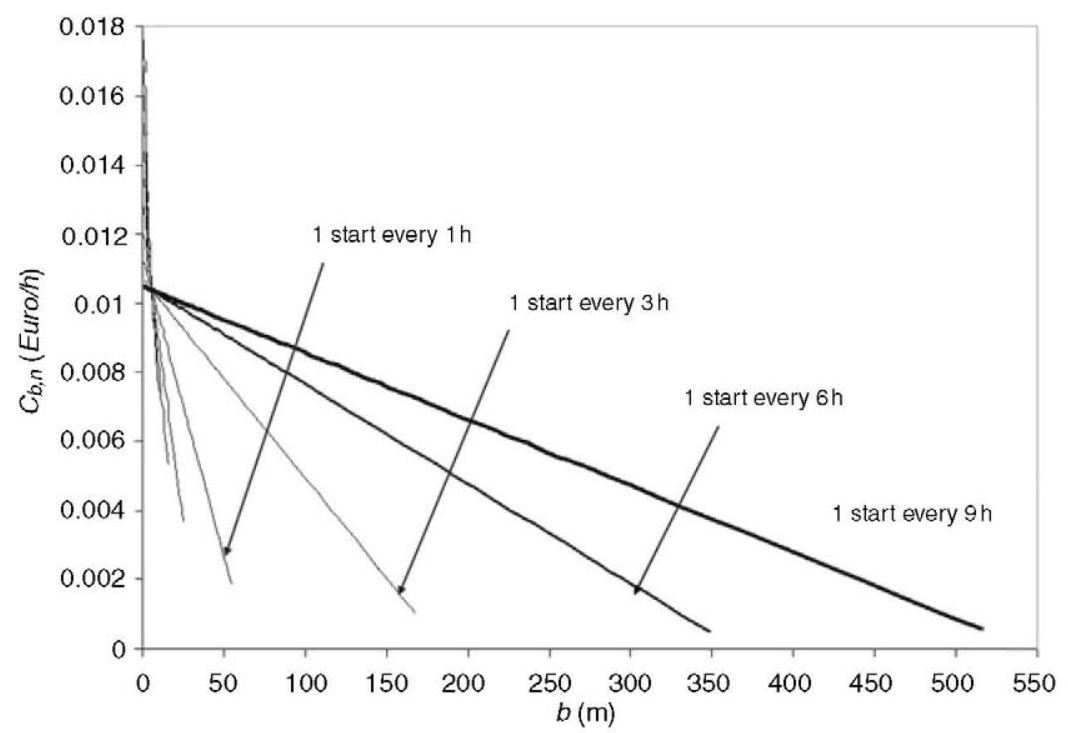

Figure 10 Cost of a compact fluorescent lamps with integrated ballasts (CFLi) manufactured in Germany or Slovakia in operation in Spain over time off

common sense dictates with regard to operating the lamp for the least time possible with the lowest number of starts.

However, the representation of emissions and cost over time $b$, the time the lamp remains off, provides additional information. Figures 9 and 10 represent the emissions of $\mathrm{Hg}$ and costs, respectively, for a CFLi manufactured in Germany and in operation in Spain over time off $b$. The families of 
curves that appear in each graph are observed to have a radial point where they cross. This point is given by Equations (15) and (20) for $\mathrm{Hg}$ and cost considering the discount rate (and in Equations (12) and (21) for $\mathrm{CO}_{2}$ and the economic cost without considering the discount rate) and represent times off $b_{o p t}^{H g}$ and $b_{o p t}$ for which $\mathrm{Hg}$ emissions and cost, respectively, are identical in any combination of $a$ and $n$. If the CFLi is operated such that it respects the optimal $b$, it at least ensures that the highest emissions cost zones are avoided, whatever the number of starts and length of $a$. If, in addition, one takes the precaution of increasing $b$ over the optimum, the emissions and costs are lowered considerably.

In the case of CFLi manufactured in Germany, the optimal $b_{\text {opt }}^{\mathrm{CO}_{2}}$ for $\mathrm{CO}_{2}$ equivalent emissions does not exceed 68 seconds, that is, all the emissions and costs lines arise from almost zero $b$. The value of $b_{o p t}^{\mathrm{CO}_{2}}$ for a CFLi manufactured in Slovakia is 34 seconds. $b_{\text {opt }}^{\mathrm{Hg}}$ is 32 minutes for $\mathrm{Hg}$ emissions (nearly the same for CFLi manufactured in Slovakia and in Germany) and $b_{o p t}$ is up to 6 minutes for the cost of the lamp. If the discount rate is not considered, $b_{\text {opt }}$ is 13 minutes. Some recommendations suggest that fluorescent lamps not be turned off for a time under 5 minutes to 15 minutes. ${ }^{17,36}$ In one case, the recommendation is based on the definition of lifetime $^{17}$ and in another case on cost reduction. ${ }^{36}$ However, there is no additional information on the calculation method.

As observed in Figure 9, there is a critical maximum $b$ for each cycle after which the cycle does not exist, since the sum of $a$ and $b$ makes the corresponding $n$ impossible.

The analysis of optimal $b$ provides a more concrete criterion than that based on the choice of $a$ or $n$. If the user is interested in reducing the environmental impact of his CFLi, he should keep in mind that it is not a good idea to turn the lamp off if he plans to turn it on again before $b_{o p t}^{H g}$. If the user wishes to lower the cost of his CFLi, he should keep in mind that it is inadvisable to turn off the lamp, if he plans to turn it on again before $b_{\text {opt }}$. If he uses the environmental criterion under the current conditions of the $\mathrm{Hg}$ cycle an acceptable efficiency can also be reached for the economic cost.

\subsection{Effect of quality and price of the CFLi}

The above analysis is based on the use of a CFLi of reasonable quality, and price and quality similar to those of the major brands. However, there are a multitude of products of doubtful quality at a much lower price than have been considered up to now.

This section analyzes the effect on lamp lifetime of assuming different times of turning it on and off using different $e$ and $f$, respectively, and lower purchase prices. Figure 1 shows the lifetime of the CFLi considered over $a$. The $a$ at which the calculations are made are also shown. Table 2 shows $e$ and $f$, the purchase prices considered and the names of the CFLi models proposed: G, B and VB (good, bad and very bad, respectively). The prices of poor-quality CFLi (B and VB) are typical of presumably low quality that may be found in super centres and low-cost product stores in Madrid. As in the case of Model G, the poor-quality models do not correspond to specific products found on the market. It is possible for low-price, good-quality models to be found and vice versa. However, practical experience of the authors as users and of other users consulted suggests that it is more likely to find low-price, low-quality models than the contrary.

The Hg content in the gas in the lamp and the electricity used in its manufacture is still as shown in Table $1 . \mathrm{CO}_{2}$ and $\mathrm{Hg}$ emissions during manufacturing take Germany as a reference. However, it is assumed that lowquality models may also worsen both the energy used in manufacturing them and their $\mathrm{Hg}$ content, while the price of labour is very low. 
Emissions and costs and optimal time off for a selection of cycles are analyzed: Cycle A with 545 on/15 off, Cycle B with 165 on/375 off, Cycle B' with 165 on/15 off, Cycle $C$ with 45 on/15 off, Cycle D with 15 on/15 off and Cycle $\mathrm{E}$ with $5 / 5$, which provides an idea of the effect of starts and operating time in specific cases. Figure 1 shows the values of $a$ mentioned. Cycle $\mathrm{B}^{\prime}$ represents conventional cycling and it may be compared with Cycle B, which is continuous operation for a similar time but with long intervals turned off. Cycle E corresponds to very short periods of on and off. The problem with this type of cycling ${ }^{37,38}$ is that the lifetime of the lamp is hard to predict: It is possible for Equation (4) not to be able to describe the lifetime of the CFLi adequately. The analysis is included because it is considered that the lifetime model is the best estimate available and to characterize what could happen in the extreme case of using the CFLi in places with traffic where users with a sense of saving turn it on and off very frequently.

Table 4 shows the $\mathrm{CO}_{2}\left(E_{a, n}^{\mathrm{CO}_{2}}\right)$ and $\mathrm{Hg}\left(E_{a, n}^{\mathrm{Hg}}\right)$ emissions and the cost $\left(C_{a, n}\right.$ computed for $T_{e}=3$ years and $i=7 \%$ ) as result of introducing the values of the variables (in particular Cycles A, B, B', C, D and E) in Equations (10), (13) and (17). It also shows the ratio of maximums and minimums (Max/Min row) for each model of CFLi and for each operating cycle considered (Max/Min column). Max/ Min row correspond to the rows in the table giving information on the sensitivity of emissions and costs of a certain CFLi to different cycles. The Max/Min column corresponds to columns in the table that give information on

Table $4 \mathrm{CO}_{2}$ and $\mathrm{Hg}$ emissions and economic cost of several different models of CFLi in a selection of cycles. The last column shows the optimal minimum time off for each emission and economic cost. The row and column labelled Max/Min shows the ratio between the worst and the best case per model in each cycle and per cycle for each model, respectively

\begin{tabular}{llllll}
$a(\mathrm{~m}) / b(\mathrm{~m})$ & & & & \\
\hline $\mathrm{A}$ & $\mathrm{B}$ & $\mathrm{B}^{\prime}$ & $\mathrm{C}$ & $\mathrm{D}$ & $\mathrm{E}$ \\
$545 / 15$ & $165 / 375$ & $165 / 15$ & $45 / 15$ & $15 / 15$ & $5 / 5$ \\
& \multicolumn{5}{r}{ Starts/number of hours (on + off) }
\end{tabular}

Starts/number of hours (on + off)

$1 \mathrm{Start} / 9 \mathrm{~h} \quad 1 \mathrm{Start} / 9 \mathrm{~h} \quad 1 \mathrm{Start} / 3 \mathrm{~h} \quad 1 \mathrm{Start} / 1 \mathrm{~h} \quad 2 \mathrm{Start} / 1 \mathrm{~h} \quad 6 \mathrm{Start} / 1 \mathrm{~h}$

\begin{tabular}{|c|c|c|c|c|c|c|c|c|}
\hline \multirow[t]{2}{*}{ Model } & \multicolumn{6}{|c|}{$E_{a n}^{C O_{2}}(\mathrm{~g} / \mathrm{h})$} & \multirow{2}{*}{$\begin{array}{l}\text { Max/Min row } \\
3\end{array}$} & \multirow{2}{*}{$\frac{b_{o p t}^{C O_{2}}(\mathrm{~m})}{1}$} \\
\hline & 12.43 & 3.78 & 11.34 & 9.45 & 6.61 & 7.53 & & \\
\hline B & 12.67 & 3.87 & 11.61 & 9.84 & 7.17 & 9.02 & 3 & 2 \\
\hline & 14.45 & 4.46 & 13.39 & 11.79 & 9.40 & 14.01 & 3 & 5 \\
\hline Max/Min column & 1.2 & 1.2 & 1.2 & 1.2 & 1.4 & 1.9 & & \\
\hline Model & \multicolumn{6}{|c|}{$E_{a n}^{H g}(\mathrm{ng} / \mathrm{h})$} & Max/Min row & $b_{o p t}^{H g}(\mathrm{~m})$ \\
\hline G & 382 & 131 & 392 & 461 & 565 & 1337 & 10 & 32 \\
\hline B & 582 & 206 & 618 & 787 & 1039 & 2583 & 13 & 43 \\
\hline VB & 2075 & 703 & 2109 & 2427 & 2905 & 6764 & 10 & 30 \\
\hline Max/Min column & 5 & 5 & 5 & 5 & 5 & 5 & & \\
\hline Model & \multicolumn{6}{|c|}{$C_{a, n}(\mathrm{cE}$ uro $/ \mathrm{h})$} & Max/Min row & $b_{o p t}(\mathrm{~m})$ \\
\hline $\mathrm{G}$ & 1.058 & 0.328 & 0.984 & 0.876 & 0.715 & 1.107 & 3 & 6 \\
\hline B & 1.079 & 0.336 & 1.007 & 0.909 & 0.763 & 1.233 & 4 & 7 \\
\hline VB & 1.245 & 0.388 & 1.165 & 1.060 & 0.903 & 1.491 & 4 & 7 \\
\hline Max/Min column & 1.18 & 1.18 & 1.18 & 1.21 & 1.26 & 1.35 & & \\
\hline
\end{tabular}

CFLi: compact fluorescent lamps with integrated ballast; Hg: mercury. 
the sensitivity of emissions and costs in a certain cycle to the quality of CFLi used.

It is observed in the Max/Min rows in Table 4 that the use of one cycle or another has a strong influence on emissions and costs for any quality of lamp. Multiplication factors of three and four were found for $\mathrm{CO}_{2}$ emissions and cost. That is, the cycles with the highest $\mathrm{CO}_{2}$ emissions and the highest costs may triple or quadruple emissions and costs. The $\mathrm{Hg}$ emissions are even more dramatic, since the selection of the cycle can multiply $\mathrm{Hg}$ emissions up to 10 to 13 times.

For a same cycle, the Max/Min columns in Table 4 show that the sensitivity to CFLi quality is rather lower than the choice of cycle. However, the effect is not in the least negligible and the choice of a poor-quality CFLi can raise $\mathrm{CO}_{2}$ emissions from $16 \%$ to $86 \%$, costs from $18 \%$ to $35 \%$ and $\mathrm{Hg}$ emissions can be multiplied five times.

The most important factor in lowering $\mathrm{CO}_{2}$ equivalent emissions is using the proper cycle, which in the examples in Table 4 lead to an increase of up to three times compared to the cycle with the most emissions and the cycle with the least emissions. This is explained by the heavier weight of $\mathrm{CO}_{2}$ emissions during lamp operation than during manufacturing (Figure 3). For each cycle, although not negligible, the quality of the lamp has a secondary role. The $b_{\text {opt }}^{\mathrm{CO}_{2}}$ for which the $\mathrm{CO}_{2}$ emissions are identical regardless of cycling of a specific CFLi is relatively low up to 5 minutes for a poor-quality CFLi.

For $\mathrm{Hg}$ emissions, $E_{a, n}^{H g}$ both CFLi quality and cycling lead to very important factors in the proportion of emissions of up to 5 and 13 , respectively. In this case, the $\mathrm{Hg}$ content in the gas of the CFLi has a determining role (Figure 4), emissions skyrocket with the reduction in lifetime associated with short cycles (Cycles D and E) compared to cycles characterized by fewer starts (Cycles A, B, B' and $\mathrm{C}$ ). The quality of the CFLi also plays an important role in $\mathrm{Hg}$ emissions, multiplying
$\mathrm{Hg}$ emissions five times for the same cycle. The minimal time off $b_{\text {opt }}^{\mathrm{Hg}}$ at which the $\mathrm{Hg}$ emissions for a specific lamp are identical in any cycle are up to 30 minutes and 43 minutes. It is important to keep in mind that a relatively low $\mathrm{Hg}$ content has been assigned to the CFLi. One study ${ }^{25}$ shows that there are CFLi models with a much higher $\mathrm{Hg}$ content than used in this one. It is reasonable to assume that such cases correspond to lower quality, lower priced models and higher $e$ and $f$. Under these assumptions, the $\mathrm{Hg}$ emissions skyrocket over what appears in Table 4.

The cost of operation of the CFLi, $C_{a, n}$ is similar to $\mathrm{CO}_{2}$ emissions. The choice of cycle can multiply the cost by four, instead of by three as in the case of $\mathrm{CO}_{2}$. The reason is that the purchase price has a weight comparable to the operating cost, at least in cycles with $a$ below 7 minutes (Figure 5). The minimum time off $b_{\text {opt }}$ at which the CFLi costs are identical regardless of cycling is from $6 \mathrm{~min}$ utes to 7 minutes depending on the quality of the CFLi. Figure 11 shows the influence of assuming different discount rates and periods of calculation for Model $\mathrm{G}$, giving values of $b_{\text {opt }}$ in the range 2 minutes to 14 minutes. If the discount rate is not considered, the $b_{\text {opt }}$ found is 13 minutes. This coincides with prior information available. ${ }^{17,36}$

Again, the reduction in polluting emissions and costs of manufacture and use of the CFLi require the minimum possible number of starts and number of hours of operation to satisfy the demand for light. This conclusion is obvious and is common sense; there is no general basic rule to orient the user based on the number of starts or the time the lamp has to remain on because Equations (10), (13) and (16) contain terms that are proportional to a and $n$.

A more specific criterion for deciding the best cycle for a certain CFLi is given by the optimal time off, which is different for the two polluting emissions considered and for the cost of the lamp. If this is respected, it at least 


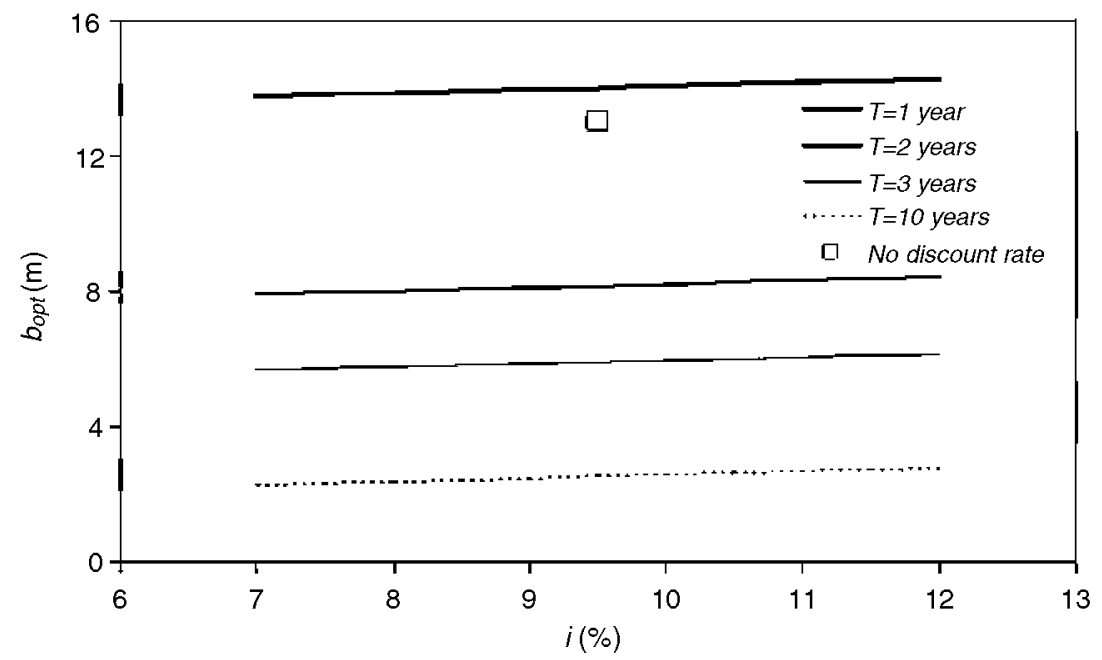

Figure 11 Optimal time off for model $G$ over the discount rate and for different calculation periods. The figure not including discount rate is also shown

ensures that the cost of generating light is identical in any cycle. If, in addition, $b$ is higher than $b_{o p t}^{\mathrm{Hg}}, b_{\text {opt }}^{\mathrm{CO}_{2}}$ and $b_{\text {opt }}$, it can be ensured that the emissions and cost diminish with respect to the optimum as shown in Figures 9 and 10. It is important to observe that the information available up to now and based on economic considerations advises that it be left off from 5 minutes to 15 minutes. ${ }^{17,36}$ This study found that this is true for the economic cost, while reduction in $\mathrm{Hg}$ emissions requires noticeably higher $b$ of at least 30 minutes to 43 minutes depending on the quality of the CFLi.

Finally, as the luminous flux of the CFLi considered is $1000 \mathrm{~lm}$, a simple division of the emissions and costs of Table 4 by 1000 supplies, an estimation of the emissions and costs per lumen at each cycle. The analysis of the results is identical to that performed above. As stated in "Equations describing emissions and costs", we do not consider that this quantity supplies rules of use for CFLi suitable for the final user and an estimation of the effect of lumen maintenance have not been performed in the present work.

\subsection{Time frame of validity of results}

Contaminating emissions in the three countries included, purchase price and electricity in Spain, manufacturing recycle rate, $\mathrm{Hg}$ content of the CFLi and discount rate that appear in this study will all indubitably undergo temporary changes. For example, it might be mentioned that in super centres, there may be sales on packs of two or three good-quality lamps at a price per unit two or three times lower than the one used for the economic calculations made in this study. It should therefore be taken into account that the calculations made and the conclusions they lead to provide results that are approximately valid only at the present time, and provide criteria and methods that allow the concrete values to be updated to the time it is intended to consider.

The optimal $b$ calculated makes CFLi use criteria possible, which if publicized and applied are useful even considering any reduction in carbon content of the electricity mix, reduction of energy in manufacturing the lamps, reduction in purchase price or 
reduction in $\mathrm{Hg}$ content in the CFLi and electricity mix.

\section{Conclusions}

Evaluation of polluting emissions and economic cost of the CFLi should take the time the lamp remains on and off, how often it is turned on and its quality into account. Optimization of operating time and the number of starts requires specific calculation to be made. The basic criteria must be to lower the number of hours of operation and the number of starts, as well as the choice of a good-quality CFLi.

$\mathrm{CO}_{2}$ and $\mathrm{Hg}$ emissions, purchase price and operation of CFLi depend on lamp quality and cycling. This is especially applicable to $\mathrm{Hg}$ emissions which may vary widely depending on the quality of the model used and especially on the cycling the lamp is subjected to.

The analysis shows that for any values of $a$ and $n$ there are values of $b$ for which the cost and emissions are always the same for a specific CFLi. The practical implication for the final user of CFLi is that the user can choose to reduce cost and emissions by increasing $b$ - the time the lamp is off - over the calculated values. A specific criterion is given by the minimum time the CFLi has to remain off before turning it on again, and which in this study is called the optimal $b$. In the case of the models proposed, optimal $b$ is rather low for $\mathrm{CO}_{2}$ emissions (up to 5 minutes), higher for the cost of the CFLi (up to 7 minutes) and even higher for $\mathrm{Hg}$ emissions (up to 43 minutes). The higher $b$ is the lower emissions and costs will be. It is therefore advisable not to turn on a CFLi sooner than 43 minutes from the last time it was turned off.

Of the three parameters studied, $\mathrm{CO}_{2}$ emissions, $\mathrm{Hg}$ emissions and cost, the second is the most sensitive to cycling and CFLi quality. Implementation and improvement of $\mathrm{CFLi}$ recycling procedures and reduction in the amount of $\mathrm{Hg}$ in the lamps have the most important role in reducing $\mathrm{Hg}$ emissions during $\mathrm{CFLi}$ use. These improvements make it possible to reduce the relationship of $\mathrm{Hg}$ content in the gas in the lamp plus what is emitted during fabrication and what is emitted during lamp operation.

\section{Acknowledgement}

The authors wish to thank Deborah Fuldauer for the English translation.

\section{References}

1 Carriere LA, Rea MS. Economics of switching fluorescent lamps. IEEE

Transactions on Industry Applications 1988; 24 : 370-379.

2 García Rosillo F, Chenlo F, Fabero F. Performance and reliability of fluorescent lamps and ballast for standalone PV systems: 12th European Photovoltaic Solar Energy Conference, The Netherlands, 1994; 1674-1777.

3 Pfeifer RP. Comparison between filament lamps and compact fluorescent lamps. The

International Journal of Life Cycle Assessment 1996; 1: 8-14.

4 Balachandra P, Shekar GL. Energy technology portfolio analysis: an example of lighting for residential sector. Energy Conversion and Management 2001; 42: 813-832.

5 Topalis FV, Kostic MB, Radakovic ZR. Advantages and disadvantages of the use of compact fluorescent lamps with electronic control gear. Lighting Research and Technology 2002; 34: 279-288.

6 Mahlia TMI, Said MFM, Másjuki HH, Tamjis MN. Cost-benefit analysis and emission reduction of lighting retrofits in residential sector. Energy and Buildings 2005; 37: 573-578.

7 Parsons D. The environmental impact of compact fluorescent lamps and incandescent lamps for Australian conditions. International Journal of Life Cycle Assessment 2009; 14 : 175-183. 
8 Ramroth L. Comparison of Life-Cycle Analyses of Compact Fluorescent and Incandescent Lamps Based on Rated Life of Compact Fluorescent Lamp. Rocky Mountain Institute, Boulder, Colorado, 2008. Retrieved 22 November 2011, from http://www.p2pays. org/ref/47/46011.pdf.

9 OSRAM Opto Semiconductors GMBH. Siemens Corporate Technology. Life cycle assessment of illuminants. A comparison of light bulbs, compact fluorescent lamps and LED lamps. Executive summary, 2009.

Retrieved 22 November 2011, from http:// www.osram-os.com/osram_os/EN/About_Us/ We shape the future of light/ Our_obligation/LED_life-cycle_assessment/ OSRAM_LED_LCA_ Summary_November_2009.pdf.

10 European Lamp Companies Federation. Environmental aspects of lamps 2009. Retrieved 22 November 2011, from http://www. elcfed.org/documents/090811_ELC\%20 brochure $\% 20$ on $\% 20$ environmental $\% 20$ aspects $\% 20$ of $\% 201 \mathrm{amps}$ _updated FINAL.pdf.

11 Bakri SNS, Ramásamy RK, Surif S. Life cycle assessment of magnetic and electronic ballast for 36-W fluorescent lamp. International Journal of Life Cycle Assessment 2010; 15: 837-841.

12 Houria A, El Khouryb P. Financial and energy impacts of compact fluorescent light bulbs in a rural setting. Energy and Buildings 2010; 42: 658-666.

13 Mahlia TMI, Razak HA, Nursahida MA. Life cycle cost analysis and payback period of lighting retrofit at the University of Malaya. Renewable and Sustainable Energy Reviews 2011; 15: 1125-1132.

14 Welza T, Hischier R, Hiltya L. Environmental impacts of lighting technologies - life cycle assessment and sensitivity analysis.

Environmental Impact Assessment Review 2011; 31: 334-343.

15 Spezia CJ, Buchanan S. Maximizing the economic benefits of compact fluorescent lamps. Journal of Industrial Technology 2011; 27: $1-11$.

16 Van Tichelen P, Mudgal S, Turunnen L, Tinetti B, Thornton A, Kofod C,
Vanhooydonck L. L19: domestic lighting. Preparatory studies for ecodesign requirements of EuPs. Belgium: VITO, 2009. Retrieved 22 November 2011, from http://www.eup4light.net/assets/pdffiles/Final_part1_2/ EuP_Domestic_Part1en2_V11.pdf.

17 OSRAM. Extended life by switching off. Retrieved 22 November 2011, from http:// www.osram.com/osram_com/Tools_\%26 Services/Training_\%26_Knowledge/FAQ/ General_Lighting/Fluorescent_lamps/ index.html\#answ726.

18 Waymouth JF. Electric Discharge Lamps. Cambridge, MA: MIT Press, 1974.

19 Rosillo FG, Martin N, Egido MA.

Comparison of conventional and accelerated lifetime testing of fluorescent lamps. Lighting Research and Technology 2010; 42: 243-259.

20 Rosillo FG, Martin N, Egido MA. Prediction of fluorescent lamp lifetimes with accelerated testing. Lighting Research and Technology 2010; 42: 467-478.

21 O'Rourke C, Figueiro MG. Long-term performance of screwbase compact fluorescent lamps. Journal of the Illuminating Engineering Society 2001; 30: 30-39.

22 OSRAM. The light bulb is disappearing, but not its light. The OSRAM DULUX ${ }^{\circledR}$ retail range of energy-savinglamps. Retrieved 22 November 2011, from http://www.osram.com/ global/pdf/Consumer/General_Lighting/ Energy-Saving_lamps/ 1A2W001GB_CFLi_Retail.pdf.

23 USHIO. Product catalog. Retrieved 22 November 2011, from http://www.ushio.com/ Files/catalog/GLcatalog58-59.pdf.

24 European Lamp Companies Federation. Round Robin Test Report. Mercury Determination in Fluorescent Lamps. The Netherlands, 2010. Retrieved 22 November 2011, from http:/www.elcfed.org/documents/ Round \%20Robin\%20Test $\%$ 20Report \%201\%2002.pdf.

25 Santos EJ, Herrmanna AB, Vieiraa F, Satie C, Barreiros Q, Tatiane A, Tormenb L, Curtiusb A. Determination of $\mathrm{Hg}$ and $\mathrm{Pb}$ in compact fluorescent lamp by slurry sampling inductively coupled plasma optical emission spectrometry. Microchemical Journal 2010; 96: $27-31$. 
26 Energy Start. Environmental Protection Agency and U.S Department of Energy Program. Retrieved 22 November 2011, from http://www.energystar.gov/ia/partners/promotions/change light/downloads/ Fact Sheet $\bar{M}$ ercury.pdf.

27 General Electric. Is it true that CFLs contain mercury? Why and how much? Retrieved 22 November 2011, from http://www.gelighting.com/na/home_lighting/ask_us/ faq_compact.htm\#mercury.

28 OSRAM. Reducing_mercury. Retrieved 22 November 2011, from http://www.osram.com/ osram_com/About_Us/Sustainability/ Products/Sustainability_criteria/ Key_Performance_Indicators/Mercury/ Reducing_mercury/index.html.

29 OSRAM. Product life cycle analysis. Retrieved 23 March 2011, from http://www.osram.com/ osram_com/About_Us/Society_and the Environment_Global_Care/Production_ and the environment/Data on the environment/Product_life_cycle_analysis/ index.html.

30 Philips. Tarifa de lámparas y equipos. Febrero 2011 España. Retrieved 22 November 2011 , from http://www.lighting.philips.es/connect/ tools_literature/.

31 Commission Regulation (EC) No 244/2009 of 18 March 2009 implementing Directive 2005/ 32/EC of the European Parliament and of the Council with regard to ecodesign requirements for non-directional household lamps. Offical Journal of the EU, issue L76 of 24 March 2009, pp 3-16.
32 OSRAM. 100 years of OSRAM - light has a name. BRAND CENTENARY 2006.

Retrieved 22 November 2011, from http:// www.osram.com/osram com/About Us/ The Company/History/ E100̄Jahre_72_dpi2\%5B1\%5D.pdf.

33 Bertoldo P, Bornás D, Monni S, Piers R. How to Develop a Sustainable Energy Action Plan $(S E A P)$. JRC Scientific and Technical Reports Guidebook. Luxembourg, European Union, 2010. Retrieved 22 November 2011 , from http://publications.jrc.ec.europa.eu repository/bitstream/111111111/14204/1/ com $\% 20$ guidebook $\% 20 j \mathrm{jrc} \% 20$ format.pdf.

34 Asociación para el reciclaje de lámparas. AMBILAMP. Memoria 2008. Retrieved 22 November 2011, from http://www.ambilamp.es/sites/default/files/memoria2008 $0 . p d f$.

35 Foro Nuclear. Energía 2010. Madrid 20010. Retrieved 31 March 2011, from http:// www.foronuclear.org/energia2010-0.jsp.

36 Department of Energy of the US. Fluorescent lighting. Retrieved 22 November 2011, from http://www.energysavers.gov/your_home/ lighting daylighting/index.cfm/ mytopic $=12280$.

37 Hammer EE. Comparative starting operation characteristics of typical F40 systems. Journal of the Illuminating Engineering Society 2001; 30: 189-197.

38 Chondrakis NG, Topalis FV. Influence of cold starting on the life of T5 fluorescent tubes and $C F L$ : Proceedings of IECON. Portugal, Porto, 2009; 3566-3572. 